INTERNATIONAL JOURNAL FOR NUMERICAL METHODS IN ENGINEERING

Int. J. Numer. Meth. Engng 2008; 00:1-30

Prepared using nmeauth.cls [Version: 2000/01/19 v2.0]

\title{
Classical and enriched finite element formulations for Bloch-periodic boundary conditions
}

\author{
N. Sukumar ${ }^{1, *}$ and J. E. Pask ${ }^{2}$ \\ 1 Department of Civil and Environmental Engineering, University of California, Davis, CA 95616. \\ 2 Condensed Matter and High Pressure Physics Division, Lawrence Livermore National Laboratory, \\ Livermore, CA 94550.
}

SUMMARY

In this paper, classical and enriched finite element formulations to impose Bloch-periodic boundary conditions are proposed. Bloch-periodic boundary conditions arise in the description of wave-like phenomena in periodic media. We consider the quantum-mechanical problem in a crystalline solid, and derive the weak formulation and matrix equations for the Schrödinger and Poisson equations in a parallelepiped unit cell under Bloch-periodic and periodic boundary conditions, respectively. For such second-order problems, these conditions consist of value- and derivative-periodic parts. The value-periodic part is enforced as an essential boundary condition by construction of a value-periodic basis, whereas the derivative-periodic part is enforced as a natural boundary condition in the weak formulation. We show that the resulting matrix equations can be obtained by suitably specifying the connectivity of element matrices in the assembly of the global matrices or by modifying the Neumann

\footnotetext{
${ }^{*}$ Correspondence to: N. Sukumar, Department of Civil and Environmental Engineering, University of California, One Shields Avenue, Davis, CA 95616. E-mail: nsukumar@ucdavis.edu

Contract/grant sponsor: Department of Energy; contract/grant number: DE-AC52-07NA27344
}

Copyright (c) 2008 John Wiley \& Sons, Ltd. 
matrices via row and column operations. The implementation and accuracy of the new formulation is demonstrated via numerical examples for the three-dimensional Poisson and Schrödinger equations using classical and enriched (partition-of-unity) higher-order finite elements. Copyright (c) 2008 John Wiley \& Sons, Ltd.

KEY WORDS: Floquet waves, quantum mechanics, periodic boundary conditions, constraint equations, higher-order finite elements, partition of unity, enrichment

\section{INTRODUCTION}

The understanding of wave-like phenomena in periodic structures is of broad interestharmonic elastic waves in composite materials [1], scattering of water waves over periodic topography [2], photonic band-gap structures [3-5], and the band structure of crystalline solids [6] are notable examples. From a computational viewpoint, the relevant governing equation(s) are solved in a unit cell subject to Bloch-periodic boundary conditions (which reduce to periodic when the wavevector is zero). In acoustic wave propagation, the term Floquet conditions is often used, which is synonymous with Bloch conditions.

The finite element (FE) method is widely used to model wave-like phenomena. For unit cell models with periodic boundary conditions, there are extensive finite element applications; however, the problem of imposing Bloch-periodic boundary conditions within a finite element variational formulation has not received significant attention in finite element monographs or the wider scientific literature. Finite element methods with Bloch-periodic boundary conditions for electromagnetic field computations were treated in References [7-10]. In Reference [8], Bloch-periodic boundary conditions were incorporated via a matrix transformation, whereas equivalent row and column operations on the system matrices were employed in Reference [9]. 
Mias and co-workers [10] presented the variational formulation for the Maxwell equations subject to Bloch-periodic boundary conditions. In quantum mechanics, Hermansson and Yevick [11] used higher-order FE basis functions for band-structure calculations. Absolute errors in the four lowest bands for the $\Gamma$-point (wavevector $\mathbf{k}=\mathbf{0}$ ) energies were reported (similar accuracy for $\mathbf{k} \neq \mathbf{0}$ was noted), with the FE method requiring many more degrees of freedom than standard Fourier based methods. Ferrari [12] used the form $\psi(\mathbf{x})=u(\mathbf{x}) e^{i \mathbf{k} \cdot \mathbf{x}}$ in the construction of a weak formulation for the cell-periodic function $u(\mathbf{x})$, allowing periodic rather than Bloch-periodic trial and test spaces. Pask and co-workers [13-15] considered the required value- and derivative-periodic boundary conditions in a general unit cell to systematically derive the weak formulation and matrix equations for the Poisson and Schrödinger equations. Following the formulation of Pask et al. [13], Jun [16] developed a meshfree formulation allowing full Brillouin zone (k-point) sampling and general unit cells for crystalline solids.

In this paper, we present a simple and efficient means to impose Bloch-periodic boundary conditions in classical and enriched finite element formulations. We consider the quantummechanical problem in a crystalline solid, and derive the weak formulation and matrix equations for the Schrödinger and Poisson equations in a parallelepiped unit cell under Blochperiodic and periodic boundary conditions, respectively. For such second-order problems, these conditions consist of value- and derivative-periodic parts. The value-periodic part is enforced as an essential boundary condition by construction of a value-periodic basis, whereas the derivative-periodic part is enforced as a natural boundary condition in the weak formulation. We show that the resulting matrix equations can be obtained by specifying the connectivity of element matrices consistent with a Bloch-periodic basis in the assembly of the global matrices 
or, equivalently, by modifying the standard Neumann matrices via row and column operations. (The system matrices prior to modifications to impose boundary conditions are referred to as Neumann matrices.) The approach we present is general, and lends itself to the incorporation of Bloch-periodic boundary conditions, periodic boundary conditions, or multipoint constraints within any standard finite element implementation.

The remainder of this paper is organized as follows. In the next section, we develop the weak forms for the Schrödinger and Poisson equations subject to Bloch-periodic and periodic boundary conditions, respectively. In Section 3, the discrete equations for FE and partition-ofunity enriched finite element (PUFE) methods are presented, and the different procedures to impose Bloch-periodic boundary conditions are described. Numerical examples for the Poisson and Schrödinger equations are presented in Section 4 for classical and partition-of-unity finite element methods. We close with a few concluding remarks in Section 5.

\section{WEAK FORMULATIONS: SCHRÖDINGER AND POISSON EQUATIONS}

Consider a parallelepiped unit cell (see Figure 1) with domain $\Omega \subset \mathbb{R}^{3}$ and primitive lattice vectors $\mathbf{a}_{i}(i=1,2,3)$. In a periodic solid, the charge density and electrostatic potential are periodic, i.e.,

$$
\begin{gathered}
\rho(\mathbf{x}+\mathbf{R})=\rho(\mathbf{x}), \\
V(\mathbf{x}+\mathbf{R})=V(\mathbf{x}),
\end{gathered}
$$

and the wavefunction $\psi$, the solution of Schrödinger's equation, satisfies Bloch's theorem

$$
\psi(\mathbf{x}+\mathbf{R})=\exp (i \mathbf{k} \cdot \mathbf{R}) \psi(\mathbf{x})
$$




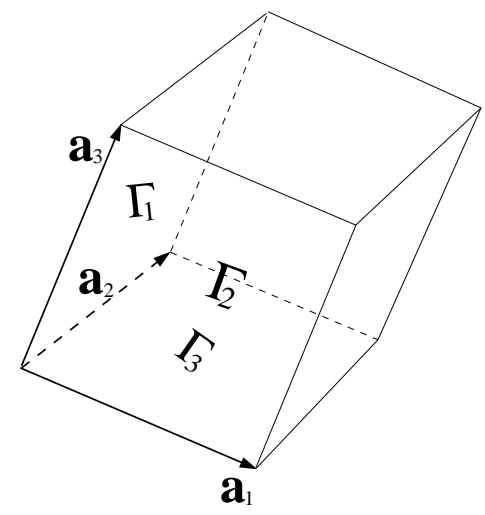

Figure 1. Parallelepiped unit cell $\Omega$, boundary $\Gamma$, and surfaces $\Gamma_{1}-\Gamma_{3}$ associated with primitive lattice vectors $\mathbf{a}_{1}-\mathbf{a}_{3}$.

where $\mathbf{R}=i_{1} \mathbf{a}_{1}+i_{2} \mathbf{a}_{2}+i_{3} \mathbf{a}_{3}\left(i_{1}, i_{2}, i_{3} \in \mathbb{Z}\right)$ is a lattice translation vector, $\mathbf{k}$ is the wavevector, and $i=\sqrt{-1}[6]$. For $\mathbf{k}=\mathbf{0}$ ( $\Gamma$-point), the wavefunction is periodic; otherwise, there is a phase shift $\exp (i \mathbf{k} \cdot \mathbf{R})$ with translation $\mathbf{R}$ from cell to cell in the solid. In such a periodic solid, the wavevector $\mathbf{k}$, also called the crystal momentum, of an electron evolves according to a Lorentz-like force law. Furthermore, $\mathbf{k}$ and $\mathbf{k}+\mathbf{G}$ are physically equivalent, where $\mathbf{G}$ is a reciprocal lattice vector, so that it is sufficient to consider just $\mathbf{k}$ in the first Brillouin zone [6].

\subsection{Schrödinger Equation}

The strong form of the Schrödinger problem in the unit cell is:

$$
\begin{gathered}
-\frac{1}{2} \nabla^{2} \psi(\mathbf{x})+V(\mathbf{x}) \psi(\mathbf{x})=\varepsilon \psi(\mathbf{x}) \quad \text { in } \Omega, \\
\psi\left(\mathbf{x}+\mathbf{a}_{\ell}\right)=\exp \left(i \mathbf{k} \cdot \mathbf{a}_{\ell}\right) \psi(\mathbf{x}) \quad \text { on } \Gamma_{\ell}, \\
\nabla \psi\left(\mathbf{x}+\mathbf{a}_{\ell}\right) \cdot \hat{\mathbf{n}}=\exp \left(i \mathbf{k} \cdot \mathbf{a}_{\ell}\right) \nabla \psi(\mathbf{x}) \cdot \hat{\mathbf{n}} \quad \text { on } \Gamma_{\ell},
\end{gathered}
$$

where $\psi$ is the wavefunction (eigenfunction), $V$ is the potential (periodic in a crystalline solid), $\varepsilon$ is the energy eigenvalue, $\mathbf{a}_{\ell}$ are the primitive lattice vectors, $\hat{\mathbf{n}}$ is the unit outward 
normal at $\mathbf{x}$, and $\Omega$ and $\Gamma_{\ell}$ are the domain and bounding surfaces shown in Figure 1 [6]. (We use atomic units throughout.) Note that, since the boundary conditions are complex-valued, the wavefunctions are in general also complex; however, due to the self-adjoint operator, the eigenvalues are real. In the ensuing derivation of the weak form, we proceed along the lines of Pask and Sterne [15]. In References [13,15], on writing $\psi$ in the form $\psi(\mathbf{x})=u(\mathbf{x}) e^{i \mathbf{k} \cdot \mathbf{x}}$, the Schrödinger equation was rewritten in terms of the periodic function $u(\mathbf{x})$ and hence periodic boundary conditions sufficed. The point of departure in this contribution is that we solve the Schrödinger equation (3a) directly for $\psi(\mathbf{x})$ satisfying conditions (3b) and (3c), rather than indirectly for periodic $u(\mathbf{x})$, and hence require Bloch-periodic rather than periodic trial and test spaces.

To construct the weak formulation of the Schrödinger problem (3a)-(3c), we take the inner product of the differential equation (3a) with an arbitrary test function $v(\mathbf{x})$, and integrate by parts (divergence theorem is invoked) to obtain

$$
\frac{1}{2} \int_{\Omega} \nabla v^{*} \cdot \nabla \psi d \mathbf{x}-\frac{1}{2} \int_{\Gamma} v^{*} \nabla \psi \cdot \hat{\mathbf{n}} d s+\int_{\Omega} v^{*}(V-\varepsilon) \psi d \mathbf{x}=0,
$$

where $v^{*}$ is the complex conjugate of $v$ and $v, \psi \in H^{1}(\Omega)$, the Sobolev space of functions square integrable in $\Omega$ up to first derivatives. To enforce the derivative boundary condition (3c), we note that since the domain is a parallelepiped, the boundary term in (4) can be written as

$$
-\frac{1}{2} \sum_{\ell} \int_{\Gamma_{\ell}}\left[v^{*}(\mathbf{x}) \nabla \psi(\mathbf{x}) \cdot \hat{\mathbf{n}}-v^{*}\left(\mathbf{x}+\mathbf{a}_{\ell}\right) \nabla \psi\left(\mathbf{x}+\mathbf{a}_{\ell}\right) \cdot \hat{\mathbf{n}}\right] d s .
$$

We now restrict $v$ to the value-periodic subspace

$$
\mathcal{V}=\left\{v \in H^{1}(\Omega): v\left(\mathbf{x}+\mathbf{a}_{\ell}\right)=\exp \left(i \mathbf{k} \cdot \mathbf{a}_{\ell}\right) v(\mathbf{x}) \text { on } \Gamma_{\ell}\right\}
$$

consistent with the value-periodic condition (3b), so that the boundary term in (4) becomes

$$
-\frac{1}{2} \sum_{\ell} \int_{\Gamma_{\ell}} v^{*}(\mathbf{x})\left[\nabla \psi(\mathbf{x}) \cdot \hat{\mathbf{n}}-\exp \left(-i \mathbf{k} \cdot \mathbf{a}_{\ell}\right) \nabla \psi\left(\mathbf{x}+\mathbf{a}_{\ell}\right) \cdot \hat{\mathbf{n}}\right] d s,
$$


which vanishes upon the assertion of the derivative-periodic condition (3c). Thus the differential equation (3a) and derivative boundary condition (3c) imply the integral relation

$$
\frac{1}{2} \int_{\Omega} \nabla v^{*} \cdot \nabla \psi d \mathbf{x}+\int_{\Omega} v^{*}(V-\varepsilon) \psi d \mathbf{x}=0 \quad \forall v \in \mathcal{V} .
$$

Conversely, it is straightforward to show by another integration by parts that the integral relation (8) implies both the differential equation (3a) and derivative boundary condition (3c). Hence, to find eigenfunctions $\psi$ and eigenvalues $\varepsilon$ satisfying (3a)-(3c) using the above relation, it remains only to satisfy the value-periodic condition (3b); and so we arrive at the following weak formulation of the problem (3a)-(3c): Find functions $\psi \in \mathcal{V}$ and scalars $\varepsilon \in \mathbb{R}$ such that

$$
\begin{gathered}
a(v, \psi)=\varepsilon(v, \psi) \quad \forall v \in \mathcal{V}, \\
a(v, \psi)=\frac{1}{2} \int_{\Omega} \nabla v^{*} \cdot \nabla \psi d \mathbf{x}+\int_{\Omega} v^{*} V \psi d \mathbf{x}, \quad(v, \psi)=\int_{\Omega} v^{*} \psi d \mathbf{x}, \\
\mathcal{V}=\left\{v \in H^{1}(\Omega): v\left(\mathbf{x}+\mathbf{a}_{\ell}\right)=\exp \left(i \mathbf{k} \cdot \mathbf{a}_{\ell}\right) v(\mathbf{x}) \text { on } \Gamma_{\ell}\right\} .
\end{gathered}
$$

In this formulation, the value-periodic condition (3b) is enforced as an essential boundary condition whereas the derivative-periodic condition (3c) is enforced as a natural one.

Since the Schrödinger operator is self-adjoint, there exists an equivalent variational formulation of the weak formulation (9): Find functions $\psi \in \mathcal{V}$ such that the Rayleigh quotient

$$
R[\psi]=\frac{a(\psi, \psi)}{(\psi, \psi)}
$$

is stationary, i.e., the first variation $\delta R[\psi]=0$. For each eigenfunction $\psi$, the corresponding eigenvalue $\varepsilon$ is given by $\varepsilon=R[\psi]$. The equivalence of the weak and the variational formulations is particularly appealing for basis-set solution methods (such as FE or PUFE) since a consequence is that the approximate eigenvalues converge monotonically from above to the exact eigenvalues as the approximation space is enlarged (minmax principle) [17]. Since the Copyright (c) 2008 John Wiley \& Sons, Ltd.

Int. J. Numer. Meth. Engng 2008; 00:1-30

Prepared using nmeauth.cls 
FE space is a subspace of the PUFE, this ensures that use of the PUFE approximation can only improve (or at worst leave unchanged) the accuracy of the eigenvalues of the Schrödinger operator in comparison to the corresponding FE solution.

\subsection{Poisson Equation}

The strong form of the Poisson problem for the electrostatic potential $V$ in the unit cell is:

$$
\begin{gathered}
-\nabla^{2} V(\mathbf{x})=4 \pi \rho(\mathbf{x}) \quad \text { in } \Omega, \\
V\left(\mathbf{x}+\mathbf{a}_{\ell}\right)=V(\mathbf{x}) \quad \text { on } \Gamma_{\ell}, \\
\nabla V\left(\mathbf{x}+\mathbf{a}_{\ell}\right) \cdot \hat{\mathbf{n}}=\nabla V(\mathbf{x}) \cdot \hat{\mathbf{n}} \quad \text { on } \Gamma_{\ell},
\end{gathered}
$$

where $\rho(\mathbf{x})$ is the charge density, which is periodic in a crystalline solid. Unlike the wavefunctions, both potential and charge density are real-valued, observable quantities. Note, however, that the only charge densities consistent with the derivative-periodic condition (10c) are those which are net neutral, i.e., $\int_{\Omega} \rho d \mathbf{x}=0$, since

$$
\int_{\Omega} \nabla^{2} V d \mathbf{x}=\int_{\Gamma} \nabla V \cdot \hat{\mathbf{n}} d \mathbf{x}=\sum_{\ell} \int_{\Gamma_{\ell}}\left(\nabla V(\mathbf{x}) \cdot \hat{\mathbf{n}}-\nabla V\left(\mathbf{x}+\mathbf{a}_{\ell}\right) \cdot \hat{\mathbf{n}}\right) d \mathbf{x},
$$

which vanishes for all $V$ satisfying (10c).

Proceeding similarly to the Schrödinger case from the previous section, we arrive at the following weak formulation of the required problem (10a)-(10c): Find $V \in \mathcal{W}$ such that

$$
\begin{gathered}
\int_{\Omega} \nabla w \cdot \nabla V d \mathbf{x}=4 \pi \int_{\Omega} w \rho d \mathbf{x} \quad \forall w \in \mathcal{W}, \\
\mathcal{W}=\left\{w \in H^{1}(\Omega): w\left(\mathbf{x}+\mathbf{a}_{\ell}\right)=w(\mathbf{x}) \text { on } \Gamma_{\ell}\right\} .
\end{gathered}
$$

As in the Schrödinger case, the value-periodic condition (10b) is enforced as an essential boundary condition whereas the derivative-periodic condition (10c) is enforced as a natural one. 


\section{DISCRETE EQUATIONS}

Having constructed weak formulations that require only value-periodicity of the trial and test spaces, i.e., conditions (10b) or (3b) for periodic or Bloch-periodic cases, respectively, we now proceed with discretization in a $C^{0}$ basis, which can be readily constructed to accommodate the required value-periodicity. Since in the present formulation the value-periodic boundary conditions (10b) and (3b) are essential whereas the derivative-periodic boundary conditions (10c) and (3c) are natural, $C^{0}$ bases that satisfy only the value-periodic conditions suffice; and so we construct and employ such bases below. In what follows, therefore, by periodic and Bloch-periodic bases, we shall mean those that satisfy the minimal value-periodicity conditions $(10 \mathrm{~b})$ and $(3 \mathrm{~b})$, respectively.

Linear (HEX8), quadratic (HEX20), and cubic (HEX32) serendipity hexahedral elements (see Figure 2) are used to discretize the parallelepiped unit cell. Shape function expressions for serendipity brick elements are given in Reference [18]. The classical finite element bases are augmented by additional functions (enriched basis functions) via the framework of partition of unity $[19,20]$. The resulting augmented (PUFE) bases are used to discretize the weak formulations.

\subsection{Poisson Equation}

The PUFE approximation (trial function) for the potential is:

$$
V^{h}(\mathbf{x})=\sum_{i \in I} \phi_{i}(\mathbf{x}) v_{i}+\sum_{\alpha} \sum_{j \in J} \phi_{j}^{\mathrm{PU}}(\mathbf{x}) \tilde{V}_{\alpha}(\mathbf{x}) a_{j \alpha} \equiv \sum_{k=1}^{N+M} \Phi_{k}(\mathbf{x}) d_{k},
$$

where $\phi_{i}(\mathbf{x})$ are FE basis functions, $\phi_{j}^{\mathrm{PU}}(\mathbf{x})$ are $\mathrm{FE}$ basis functions used to form the enriched

basis functions, $\tilde{V}_{\alpha}(\mathbf{x})$ are enrichment functions, and $v_{i}$ and $a_{j \alpha}$ are nodal coefficients associated with the finite element and enriched bases, respectively. The finite element bases are $\left\{\phi_{i}\right\}_{i=1}^{N}$, Copyright (c) 2008 John Wiley \& Sons, Ltd.

Int. J. Numer. Meth. Engng 2008; 00:1-30

Prepared using nmeauth.cls 


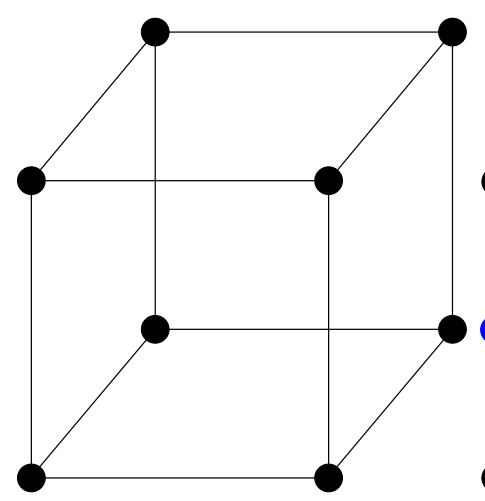

(a)

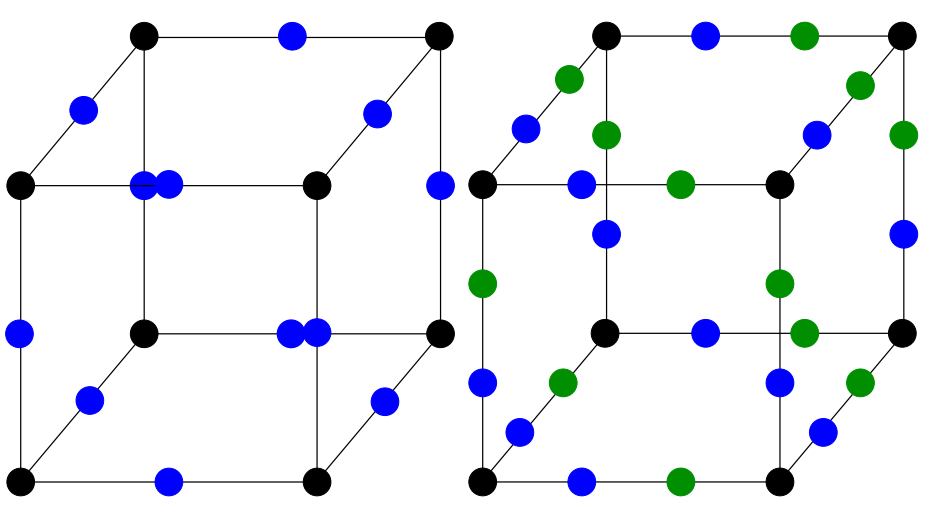

(c)

Figure 2. Higher-order serendipity brick elements. (a) Linear (HEX8); (b) Quadratic (HEX20); and (c) Cubic (HEX32).

whereas the enriched bases are $\bigcup_{\alpha}\left\{\phi_{j}^{\mathrm{PU}} \tilde{V}_{\alpha}\right\}_{j=1}^{M_{\alpha}}\left(M_{\alpha} \leq N\right)$, with $M=\sum_{\alpha} M_{\alpha}$ in (13). On substituting the above trial function and using $\Phi_{i}$ as test functions in the weak formulation (12), we obtain the following discrete system of equations:

$$
\mathbf{K d}=\mathbf{f}, \quad \mathbf{d}=[\mathbf{v} \mathbf{a}]^{T}, \quad \mathbf{K}_{i j}=\int_{\Omega} \nabla \Phi_{i} \cdot \nabla \Phi_{j} d \mathbf{x}, \quad \mathbf{f}_{i}=4 \pi \int_{\Omega} \Phi_{i} \rho d \mathbf{x}
$$

where $\Phi_{i}=\phi_{i}$ for a classical degree of freedom and $\Phi_{i}=\phi_{i}^{\mathrm{PU}} \tilde{V}_{\alpha}$ for an enriched degree of freedom. Note that due to the self-adjoint differential operator and identical test and trial spaces in the weak formulation, the resulting linear system is symmetric.

In order for the trial and test functions to be admissible in the weak formulation (12), they must span a subspace of the value-periodic subspace $\mathcal{W}$, i.e., satisfy the value-periodic boundary condition (10b). This is achieved if the FE basis $\left\{\phi_{i}\right\}$, partition of unity basis $\left\{\phi_{i}^{\mathrm{PU}}\right\}$, and enrichment functions $\tilde{V}_{\alpha}$ satisfy the condition, so that the final PUFE basis $\left\{\Phi_{i}\right\}=\left\{\phi_{j}\right\} \bigcup\left\{\phi_{j}^{\mathrm{PU}} \tilde{V}_{\alpha}\right\}$ must satisfy the condition as well. By virtue of the locality of the finite element and partition of unity basis functions, all basis functions $\Phi_{i}$ associated with interior Copyright (c) 2008 John Wiley \& Sons, Ltd.

Int. J. Numer. Meth. Engng 2008; 00:1-30 


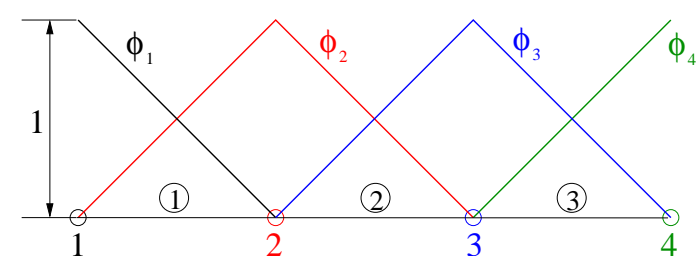

(a)

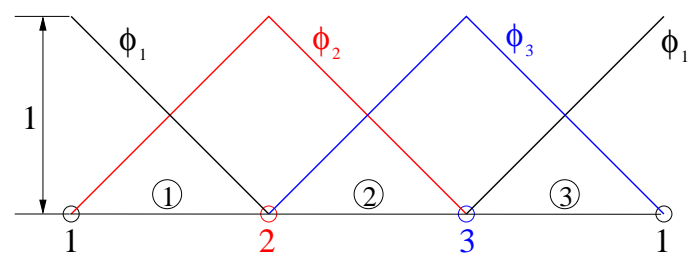

(c)

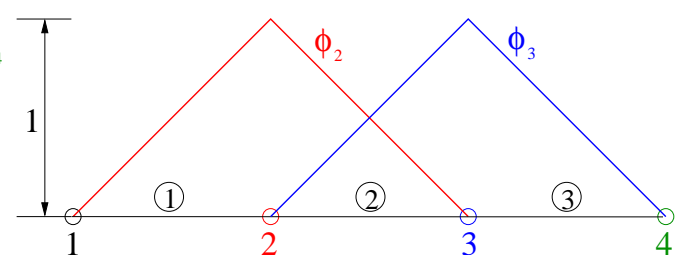

(b)

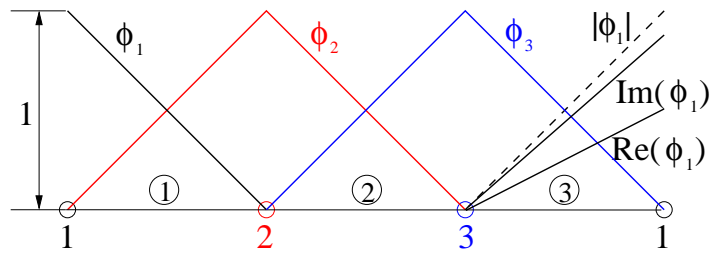

(d)

Figure 3. Linear finite element bases. (a) Neumann; (b) Dirichlet; (c) Periodic; and (d) Blochperiodic. The real and imaginary parts of $\phi_{1}$ are illustrated for the Bloch basis in (d) for phase shift $k a=\pi / 3(\Omega=[0, a])$. The modulus of $\phi_{1}$ is the same at $x=0$ and $x=a$.

nodes satisfy the required condition (10b) since they vanish on the boundary $\Gamma$. However, basis functions $\Phi_{i}$ associated with boundary nodes do not a priori satisfy (10b), and hence require special treatment.

We first consider the pure FE case. Figure 3 illustrates the basic ideas in 1D. Figure 3a shows a standard one-dimensional linear FE basis spanning the space of piecewise-linear polynomials with nodes at interelement boundaries. Such a basis is appropriate for discretization of a weak form expressed in terms of general $C^{0}$ trial and test spaces with no constraints at the boundary. Discretization in such a basis then yields solutions which satisfy the Neumann (natural) boundary conditions contained in the weak formulation. We thus refer to this as a Neumann basis. The other bases relevant to the present work can be derived from it. Figure $3 \mathrm{~b}$ shows an FE basis that vanishes at the domain boundary. It can be obtained from the Neumann Copyright (C) 2008 John Wiley \& Sons, Ltd.

Int. J. Numer. Meth. Engng 2008; 00:1-30 
basis by omitting the Neumann basis functions $\phi_{1}$ and $\phi_{4}$. Discretization in such a basis will enforce vanishing Dirichlet boundary conditions. In Figure 3c, a periodic FE basis is depicted. It can be obtained from the Neumann basis by combining Neumann basis functions $\phi_{1}$ and $\phi_{4}$ to form periodic basis function $\phi_{1}$. Discretization in such a basis will enforce the value-periodic boundary condition (10b) and so this is precisely the kind of basis required for discretization of the Poisson weak formulation (12). The extension to higher-dimensions is straightforward: to construct a periodic FE basis in a general parallelepiped unit cell $\Omega$, we combine all boundary basis functions related by a lattice translation vector $\mathbf{R}$.

Use of constraint equations and ensuing transformations of the stiffness matrix, and penalizing via Lagrange multipliers are commonly used to impose periodic boundary conditions [1]. Here, we enforce the required conditions (10b) and (10c) in a variationally consistent manner, via discretization of the weak formulation (12) containing the derivative condition (10c) as a natural boundary condition, in a value-periodic $C^{0}$ basis spanning a subspace of the required trial/test space $\mathcal{W}$ satisfying the value-periodic condition (10b). This can be accomplished in one of two ways: (1) by enforcing nodal connectivity consistent with value-periodicity in the assembly of the global system matrices [14], or (2) via row and column operations after assembly of the usual Neumann matrices. In this section, we consider the former approach; in Section 3.2, we consider the latter, in the context of the more general Bloch-periodic conditions.

We first partition the boundary nodes into reference nodes not related to one another by a lattice translation $\mathbf{R}$ and remaining image nodes related to the reference nodes via lattice translations. For example, in Figure 3a, the reference node is node 1 and its image on the opposite boundary is node 4, which is reflected in Figure 3c by the renumbering of the image 
node. In practice, then, periodicity can be enforced during assembly simply by replacing all image node numbers by their corresponding reference node numbers in the connectivity matrices. For example, in the basis of Figure 3, the connectivity of local node 2 of element 3 is changed from global node 4 to global node 1 . We emphasize that the image nodes retain their geometric coordinates. The replacement of image node numbers by associated reference node numbers in the connectivity matrices merely enforces the continuity of the element shape functions across the domain boundary by associating shape functions at image nodes with corresponding shape functions at reference nodes, thus producing system matrices consistent with the desired global periodic basis.

If enrichment is used, then the enriched basis $\left\{\phi_{i}^{\mathrm{PU}} \tilde{V}_{\alpha}\right\}$ has the required value-periodicity (10b) if both $\phi_{i}^{\mathrm{PU}}$ and $\tilde{V}_{\alpha}$ have that periodicity. Periodic $\phi_{i}^{\mathrm{PU}}$ are constructed by modification of the associated connectivity matrices as discussed above. Periodic $\tilde{V}_{\alpha}$ are constructed via lattice summation. Since enrichment functions need not have compact support, practical computational considerations demand that they be localized so that only nearest-neighbor or second nearest-neighbor cells are sufficient in the lattice summation to reproduce the infinite sum. Consider a spherically symmetric enrichment function $\tilde{v}_{\alpha}(\mathbf{x}) \equiv \tilde{v}\left(\left|\mathbf{x}-\boldsymbol{\tau}_{\alpha}\right|\right)$ centered at position $\boldsymbol{\tau}_{\alpha}$ in the unit cell. We form the periodic enrichment function for the potential as

$$
\tilde{V}_{\alpha}(\mathbf{x})=\sum_{\mathbf{R}} \tilde{v}_{\alpha}(\mathbf{x}-\mathbf{R}) h\left(\left|\mathbf{x}-\tau_{\alpha}-\mathbf{R}\right|, r_{0}\right),
$$

where $h\left(r, r_{0}\right) \in \mathbb{R}_{+}$is a smooth, localized cut-off function and the summation is over sufficient lattice translation vectors $\mathbf{R}$ such that the infinite sum is reproduced. With periodic $\phi_{i}^{\mathrm{PU}}$ and $\tilde{V}_{\alpha}$, the enriched basis $\left\{\phi_{i}^{\mathrm{PU}} \tilde{V}_{\alpha}\right\}$ is periodic and so, in combination with periodic FE basis $\left\{\phi_{j}\right\}$, constitutes a periodic PUFE basis $\Phi_{k}$ appropriate for discretization of the weak formulation (12). 


\subsection{Schrödinger Equation}

The PUFE approximation (trial function) for the wavefunction is:

$$
\psi^{h}(\mathbf{x})=\sum_{i \in I} \phi_{i}(\mathbf{x}) c_{i}+\sum_{\alpha} \sum_{j \in J} \phi_{j}^{\mathrm{PU}}(\mathbf{x}) \tilde{\psi}_{\alpha}(\mathbf{x}) a_{j \alpha} \equiv \sum_{k=1}^{N+M} \Phi_{k}(\mathbf{x}) d_{k},
$$

where $\tilde{\psi}_{\alpha}(\mathbf{x})$ are the enrichment functions centered at positions $\boldsymbol{\tau}_{\alpha}$, and $c_{i}$ and $a_{j \alpha}$ are nodal coefficients associated with the finite element and enriched bases, respectively. (Note that in the case of multiple enrichments at a given center, the $\boldsymbol{\tau}_{\alpha}$ are not all distinct.) On substituting the above trial function and using $\Phi_{i}$ as test functions in the weak formulation (9), we obtain the following discrete generalized eigenproblem:

$$
\begin{gathered}
\mathbf{H d}=\varepsilon \mathbf{S d}, \quad \mathbf{d}=[\mathbf{c} \mathbf{a}]^{T}, \\
\mathbf{H}_{i j}=\frac{1}{2} \int_{\Omega} \nabla \Phi_{i}^{*} \cdot \nabla \Phi_{j} d \mathbf{x}+\int_{\Omega} \Phi_{i}^{*} V \Phi_{j} d \mathbf{x}, \\
\mathbf{S}_{i j}=\int_{\Omega} \Phi_{i}^{*} \Phi_{j} d \mathbf{x},
\end{gathered}
$$

where $\varepsilon$ is the energy eigenvalue, and $\mathbf{H}$ and $\mathbf{S}$ are the discrete Hamiltonian and overlap matrices, respectively. Note that due to the self-adjoint differential operator and identical trial and test spaces in the weak formulation, the resulting eigenproblem is Hermitian.

Now, for the trial and test functions to be admissible in the weak formulation (9), they must span a subspace of the Bloch-periodic space $\mathcal{V}$, i.e., satisfy the Bloch-periodic boundary condition (3b). Referring to (16), this can be accomplished in two distinct ways:

- $\phi_{i}$ and $\phi_{j}^{\mathrm{PU}}$ are Bloch-periodic, and $\tilde{\psi}_{\alpha}$ are periodic. Note that the finite element basis functions $\phi_{i}$ and $\phi_{j}^{\mathrm{PU}}$ are now complex valued.

- $\phi_{i}$ are Bloch-periodic, $\phi_{j}^{\mathrm{PU}}$ are periodic, and $\tilde{\psi}_{\alpha}$ are Bloch-periodic. Here, $\phi_{i}$ and $\tilde{\psi}_{\alpha}$ are complex valued. 
A 1D Bloch-periodic FE basis is shown in Figure 3d. It can be obtained from the standard Neumann basis in Figure 3a by multiplying the Neumann basis function $\phi_{4}$ by the Bloch phase factor $\exp (i k a)$ corresponding to its position at $x=a$, then combining it with the Neumann basis function $\phi_{1}$ to form the complex Bloch-periodic basis function $\phi_{1}$ shown in Figure 3d. Extension to 3D is straightforward as in the periodic case: all boundary basis functions at nodes $\mathbf{x}_{j}$ are multiplied by the appropriate Bloch phase $\exp \left(i \mathbf{k} \cdot \mathbf{x}_{j}\right)$, then those at image nodes are combined with those at corresponding reference nodes to form the required Bloch-periodic boundary functions.

To derive the system matrices corresponding to the discretization of the weak formulation (9) in such a Bloch-periodic basis, one could proceed as in Section 3.1 by modifying the connectivity matrices in the assembly process (with application of appropriate phase factors); however, the same matrices can also be obtained via row and column operations on the standard Neumann matrices. This follows directly from the relation of the Neumann and Bloch-periodic bases, as we now show.

Let $\hat{O}$ be a differential operator, and let $\mathbf{A}^{n}$ and $\mathbf{A}^{b}$ be the corresponding discrete Neumann and Bloch matrices, respectively, for that operator. In addition, $\Phi_{k}^{n} \equiv \Phi_{k}$ are the Neumann basis functions $(k=1,2, \ldots, N+M)$ and $\Phi_{k}^{b}$ are the Bloch basis functions $\left(k=1,2, \ldots, N_{b}\right.$; $\left.N_{b}<N+M\right)$. In Dirac notation [21], we have then

$$
\begin{aligned}
& \mathbf{A}_{k \ell}^{n}=\left\langle\Phi_{k}^{n}|\hat{O}| \Phi_{\ell}^{n}\right\rangle, \\
& \mathbf{A}_{i j}^{b}=\left\langle\Phi_{i}^{b}|\hat{O}| \Phi_{j}^{b}\right\rangle .
\end{aligned}
$$

Now, due to the compact support of the finite element basis functions, Bloch basis functions Copyright (c) 2008 John Wiley \& Sons, Ltd.

Int. J. Numer. Meth. Engng 2008; 00:1-30

Prepared using nmeauth.cls 
$\Phi_{i}^{b}$ can be expressed in terms of Neumann basis functions $\Phi_{\ell}^{n}$ as

$$
\Phi_{i}^{b}=\sum_{\ell} f_{\ell} \Phi_{\ell}^{n}, \quad f_{\ell}=\exp \left(i \mathbf{k} \cdot \mathbf{x}_{\ell}\right)
$$

where the summation is over functions at reference node $\ell=i$ and corresponding image nodes $\ell \neq i, f_{\ell}$ is the Bloch phase factor (unity for periodic boundary conditions), and $\mathbf{x}_{\ell}$ is the coordinate of node $\ell$. Hence, (20b) can be expanded as

$$
\begin{aligned}
\mathbf{A}_{i j}^{b} & =\left\langle\Phi_{i}^{b}|\hat{O}| \Phi_{j}^{b}\right\rangle \\
& =\left\langle\sum_{k} f_{k} \Phi_{k}^{n}|\hat{O}| \sum_{\ell} f_{\ell} \Phi_{\ell}^{n}\right\rangle=\sum_{k, \ell} f_{k}^{*} f_{\ell}\left\langle\Phi_{k}^{n}|\hat{O}| \Phi_{\ell}^{n}\right\rangle=\sum_{k, \ell} f_{k}^{*} f_{\ell} \mathbf{A}_{k \ell}^{n} .
\end{aligned}
$$

A sequence of row and column operations to transform Neumann system matrices to Bloch system matrices is thus as follows:

- Multiply all $\mathbf{A}_{k \ell}^{n}$ associated with boundary nodes by $f_{k}^{*} f_{\ell}$.

- To each row $i$ of $\mathbf{A}^{n}$ associated with a reference node, add all rows $k$ associated with corresponding image nodes; then to each column $j$ associated with a reference node, add all columns $\ell$ associated with corresponding image nodes.

- Delete all rows $k$ and columns $\ell$ associated with image nodes.

Note that the above sequence corresponds to periodic enrichment functions with Bloch-periodic partition. For Bloch-periodic enrichment and periodic partition, the phase factors $f_{\ell}$ associated with enriched DOFs would be unity. Note also that the above operations need be applied only to matrix elements associated with boundary nodes, leaving all others untouched. Furthermore, the number of complex matrix elements can be reduced if desired by dividing the matrix elements associated with each reference node and all its images by the Bloch phase factor associated with that reference node. This produces equivalent system matrices corresponding to all real-valued basis functions at reference nodes, as would be produced by Bloch sums [6] 
of the Neumann basis functions. Finally, we note that by virtue of commutativity, the above operations may be ordered as desired, consistent with the summation (22).

For the Schrödinger equation, this algorithm is applied to the $\mathbf{H}$ and $\mathbf{S}$ matrices and proves to be an efficient method to impose Bloch boundary conditions using simple row and column operations. In spirit, this parallels the manner in which Dirichlet boundary conditions are imposed in finite element methods. For the solution of the Schrödinger equation that arises in electronic-structure calculations, this approach presents significant advantages. In a quantummechanical calculation of a crystalline solid, the Schrödinger equation may be solved for $\mathcal{O}\left(10^{4}\right)$ k-points in the Brillouin zone. The proposed formulation and implementation requires the construction of $\mathbf{H}$ and $\mathbf{S}$ just once, and thereafter the Neumann-to-Bloch algorithm can be applied for each k-point, without need of further matrix element integrations, leading to substantial savings in computational costs when many k-points are required. If the boundary conditions are periodic $\left(f_{\ell}=1\right.$ in $\left.(21)\right)$, then a periodic matrix that is identical to that obtained via the procedure presented in Section 3.1 is obtained. With suitable modifications, the proposed algorithm can be adapted to handle multipoint constraints, which is a common need that arises in many finite element applications.

\section{NUMERICAL EXAMPLES}

We consider three benchmark three-dimensional unit cell problems to validate the present formulation and computational implementation. First, we consider the Poisson equation for a neutral charge density under periodic boundary conditions, and present the finite element and enriched finite element solutions. Then, three-dimensional quantum harmonic oscillator and Gaussian-well problems are solved to assess the accuracy of FE and PUFE formulations 
for periodic and Bloch-periodic boundary conditions, respectively. In the numerical examples, PUFE solutions for quadratic finite element meshes are presented, and $\phi_{j}^{\mathrm{PU}}(\mathbf{x})$, which are chosen to be trilinear (HEX8 brick element) finite element basis functions, are used to form the enriched bases that appear in equations (13) and (16).

\subsection{Poisson Equation with Net Neutral Charge Density}

As a benchmark problem, consider the following Poisson equation with a net neutral electronic charge density (localized charge density minus a constant background):

$$
\begin{gathered}
-\nabla^{2} V(\mathbf{x})=4 \pi \rho(\mathbf{x}) \quad \text { in } \Omega=(0,1)^{3}, \\
\rho(\mathbf{x})=g(|\mathbf{x}-\boldsymbol{\tau}|, 1 / 2)-1,
\end{gathered}
$$

subject to periodic boundary conditions. The charge density $g\left(r, r_{c}\right)$ is such that $\int g d \mathbf{x}=1$, and we choose $g$ and the potential associated with it $\left(-\nabla^{2} v=4 \pi g\right)$ as

$$
\begin{aligned}
& g\left(r, r_{c}\right)= \begin{cases}\frac{-21\left(r-r_{c}\right)^{3}\left(6 r^{2}+3 r r_{c}+r_{c}^{2}\right)}{5 \pi r_{c}^{8}}, & r \leq r_{c}, \\
0, & r>r_{c}\end{cases} \\
& v\left(r, r_{c}\right)=\left\{\begin{array}{ll}
\frac{9 r^{7}-30 r^{6} r_{c}+28 r^{5} r_{c}^{2}-14 r^{2} r_{c}^{5}+12 r_{c}^{7}}{5 r_{c}^{8}}, & r \leq r_{c} \\
\frac{1}{r}, & r>r_{c}
\end{array} .\right.
\end{aligned}
$$

The charge $g$ is centered at $\boldsymbol{\tau}=(1 / 2,1 / 2,1 / 2)$.

Since $g(r, 1 / 2)$ is spherically symmetric and is non-zero only within a radius of $1 / 2$, the charge densities $g$ centered in the neighboring cells of the infinite lattice have no contribution in the central cell $\Omega$. Hence, $\rho(\mathbf{x})$ is computed directly in just the unit cell. Since the problem posed does not have an analytic solution, the choice of a suitable enrichment remains to be 
determined. To this end, we note that

$$
\tilde{\rho}(\mathbf{x})=\sum_{\mathbf{R}} g(|\mathbf{x}-\boldsymbol{\tau}-\mathbf{R}|, 1 / 2)-g(|\mathbf{x}-\boldsymbol{\tau}-\mathbf{R}|, 1)
$$

is a good approximation to $\rho(\mathbf{x})$ and has the analytic solution

$$
\tilde{V}(\mathbf{x})=\sum_{\mathbf{R}} v(|\mathbf{x}-\boldsymbol{\tau}-\mathbf{R}|, 1 / 2)-v(|\mathbf{x}-\boldsymbol{\tau}-\mathbf{R}|, 1),
$$

which we adopt as the enrichment function. Since the function $v(r, 1 / 2)-v(r, 1)=0$ for $r \geq 1$, just nearest-neighbor cell contributions are sufficient to compute the infinite periodic sum in the central cell.

Figure $4 \mathrm{c}$ shows a plot of the periodic enrichment function $\tilde{V}(\mathrm{x})$ along the diagonal $(s=0$ to $s=\sqrt{3})$ of the cube. Since $\tilde{V}(\mathbf{x})$ is not localized inside the cell, rather than partition the enrichment function by the trilinear FE bases, we add the enrichment function itself to the basis. This is equivalent to constraining all the enriched degrees of freedom (DOFs) in (13) to be the same (only one additional unknown). For this example, the enriched approximation in (13) assumes the form

$$
V^{h}(\mathbf{x})=\sum_{i \in I} \phi_{i}(\mathbf{x}) v_{i}+\tilde{V}(\mathbf{x}) a,
$$

where $a$ is the unknown coefficient associated with the enrichment function $\tilde{V}(\mathbf{x})$.

The numerical results are presented in Figure 4. In Figure 4a, the charge density $\rho(\mathbf{x})$ is plotted along the diagonal of the unit cell, whereas in Figure $4 \mathrm{~b}$, the potential solutions for quadratic FE are illustrated. The plot of the enrichment function along the diagonal appears in Figure 4c. A convergence study is conducted for FE and enriched FE. Linear, quadratic, and cubic finite element computations are carried out on five meshes: $4 \times 4 \times 4,6 \times 6 \times 6$, $8 \times 8 \times 8,12 \times 12 \times 12$, and $16 \times 16 \times 16$. The number of DOFs for the HEX20 meshes are: 256 , 864, 2048, 6912, and 16384. The results for enriched FE are obtained on three quadratic FE Copyright (c) 2008 John Wiley \& Sons, Ltd. Int. J. Numer. Meth. Engng 2008; 00:1-30 


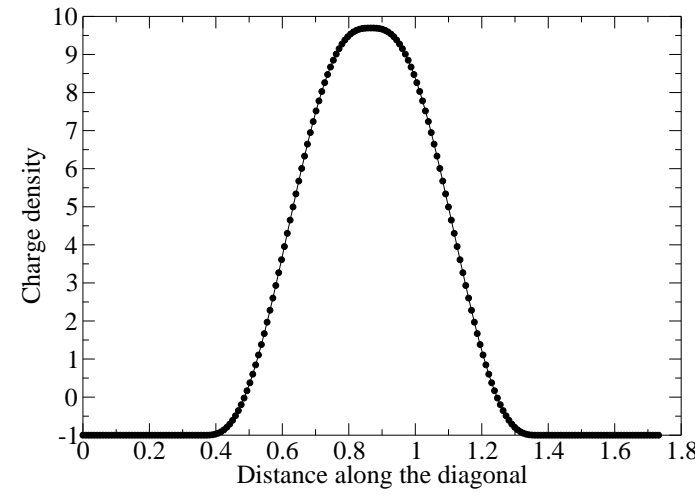

(a)

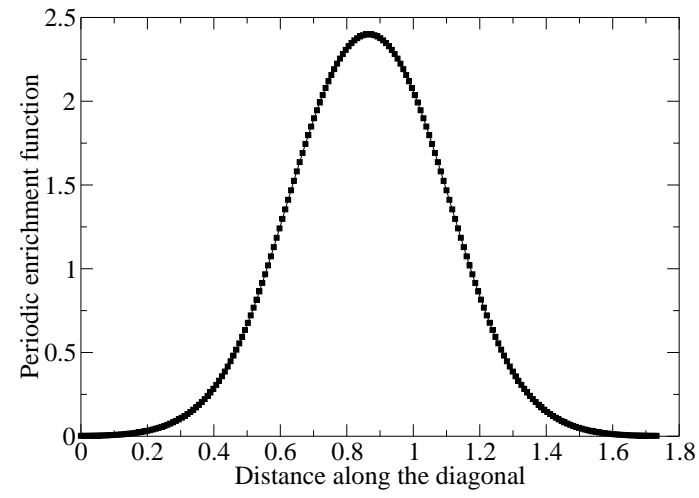

(c)

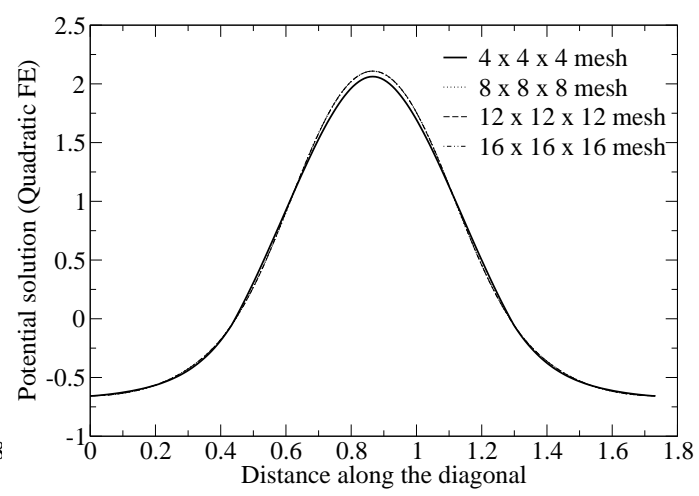

(b)

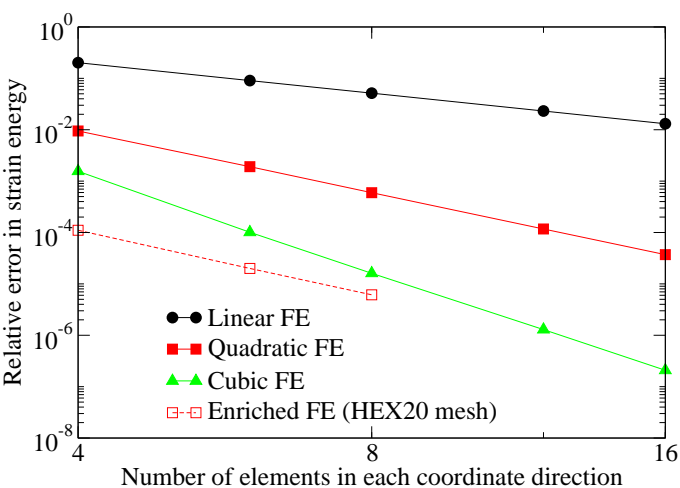

(d)

Figure 4. FE and enriched FE solutions for the Poisson problem along a diagonal of the cubic domain.

(a) Charge density $\rho(\mathbf{x})$; (b) Quadratic FE solutions; (c) Periodic enrichment function; and (d) Rate of convergence in strain energy for FE and enriched FE.

meshes $(4 \times 4 \times 4,6 \times 6 \times 6$, and $8 \times 8 \times 8)$. A $5 \times 5 \times 5$ Gauss quadrature rule is used for FE analyses, and a $8 \times 8 \times 8$ rule is adopted in enriched elements. In Figure 4 d, the relative error in the strain energy is plotted versus the number of elements in each coordinate direction. The Copyright (c) 2008 John Wiley \& Sons, Ltd.

Int. J. Numer. Meth. Engng 2008; 00:1-30 
relative error in the strain energy is defined as:

$$
E=\frac{a(V, V)-a\left(V^{h}, V^{h}\right)}{a(V, V)}, \quad a(V, V)=\int_{\Omega} \nabla V \cdot \nabla V d \mathbf{x}
$$

where $\frac{1}{2} a(V, V)$ is the exact strain energy. We use a cubic FE solution $(24 \times 24 \times 24$ mesh, 96768 DOFs) as the reference solution for $a(V, V)$. The optimal rate of convergence in strain energy is $\mathcal{O}\left(h^{2 p}\right)$ for $p$ th order finite elements [17], and the slopes in Figure $4 \mathrm{~d}(-2,-4$, and -6 for linear, quadratic, and cubic FE, respectively) are in agreement with theory. In electrostatics, rather than the strain energy, results for the Coulomb energy are usually of interest- the Coulomb energy is $a(V, V) / 8 \pi$, which can be shown by invoking the divergence theorem and Poisson's equation. Similar to quadratic FE, the slope of the enriched quadratic FE solution in Figure $4 \mathrm{~d}$ is -4 ; however, the relative error is about two orders of magnitude smaller than the corresponding quadratic FE solution.

\subsection{Schrödinger Equation}

We consider the Schrödinger equation with a harmonic potential under periodic boundary conditions $(\mathbf{k}=\mathbf{0})$ and a periodic Gaussian potential under Bloch-periodic boundary conditions $(\mathbf{k} \neq \mathbf{0})$.

4.2.1. Three-dimensional quantum harmonic oscillator As a benchmark problem, we consider the Schrödinger equation given in (3a) with harmonic potential $V(|\mathbf{x}-\boldsymbol{\tau}|)=m \omega^{2}|\mathbf{x}-\boldsymbol{\tau}|^{2} / 2$, under periodic boundary conditions. Note that the potential itself is not periodic. In the computations, we set $m=\omega=1$ so that the potential becomes $V(|\mathbf{x}-\boldsymbol{\tau}|)=|\mathbf{x}-\boldsymbol{\tau}|^{2} / 2$. The 
unit cell is a triclinic box, and the primitive lattice vectors and potential center are

$$
\begin{aligned}
\mathbf{a}_{1} & =a(1.00,0.02,-0.04), \\
\mathbf{a}_{2} & =a(0.06,1.05,-0.08), \\
\mathbf{a}_{3} & =a(0.10,-0.12,1.10), \\
\tau & =\frac{\mathbf{a}_{1}+\mathbf{a}_{2}+\mathbf{a}_{3}}{2},
\end{aligned}
$$

with lattice parameter $a=5.7$ bohr. The energy eigenvalues and eigenfunctions in an infinite box, for which an analytic solution is available, are indexed by the quantum numbers $n, \ell$, and $m$ ( $m$ takes on values from $-\ell$ to $\ell$, which correspond to the $2 \ell+1$ degenerate states) [22]. The eigenvalues of the 1D harmonic oscillator in an infinite box are $n+1 / 2(n=0,1, \ldots)$; eigenvalues for the three-dimensional oscillator are sums of the one-dimensional eigenvalues. The ten lowest states for the 3D harmonic oscillator correspond to: $n=0, \ell=0$ (degeneracy of $1, \varepsilon=3 / 2$ ); $n=0, \ell=1$ (degeneracy of $3, \varepsilon=5 / 2$ ); $n=0, \ell=2$ (degeneracy of 5 , $\varepsilon=7 / 2$ ); and $n=1, \ell=0$ (degeneracy of $1, \varepsilon=7 / 2$ ). The sum of the lowest ten eigenvalues in an infinite box is $30 \mathrm{Ha}$. Under periodic boundary conditions, the sum of the lowest ten eigenvalues in the unit cell (26) is $29.892615 \mathrm{Ha}$ (reference is a $56 \times 56 \times 56$ cubic FE solution).

The enrichment functions used in the PUFE solution for the finite box consist of lattice sums of the infinite box eigenfunctions $\psi_{n \ell m}(\mathbf{x})=R_{n \ell}(r) Y_{\ell m}(\theta, \phi)$, where $R_{n \ell}(r)$ is the radial part and $Y_{\ell m}(\theta, \phi)$ are spherical harmonics [21]. We enrich each of the lowest ten states by an enrichment function. For the three-dimensional problem in an infinite box, the eigenfunctions are known analytically [22]. The lowest two (unnormalized) radial functions are

$$
R_{00}(r)=\exp \left(-r^{2} / 2\right) \quad \text { and } \quad R_{01}(r)=r \exp \left(-r^{2} / 2\right)
$$

Second nearest-neighbor cell summations are sufficient to construct the periodic enrichment Copyright (c) 2008 John Wiley \& Sons, Ltd.

Int. J. Numer. Meth. Engng 2008; 00:1-30

Prepared using nmeauth.cls 


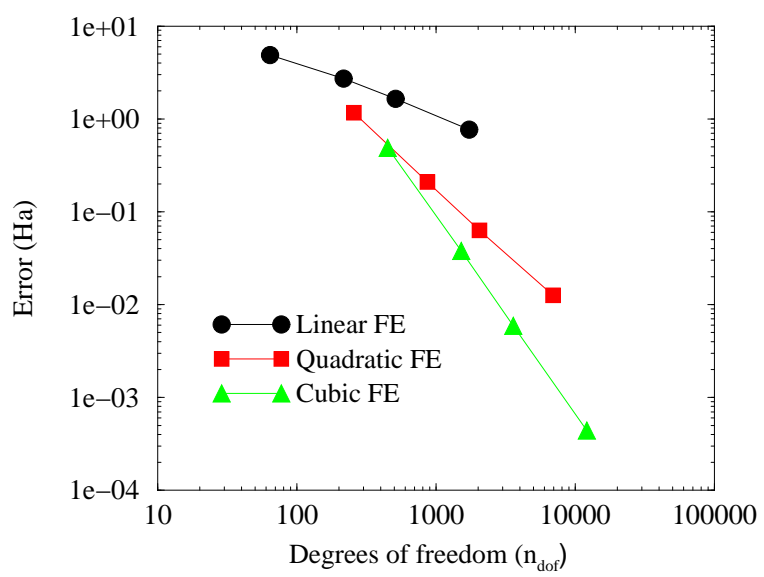

(a)

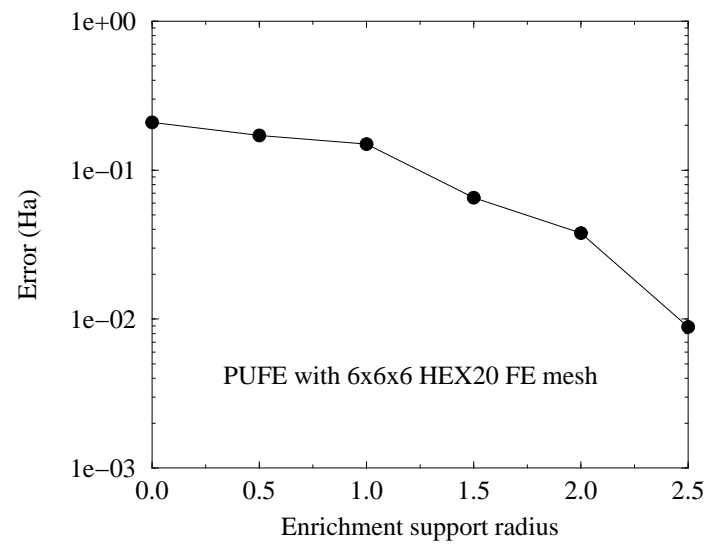

(b)

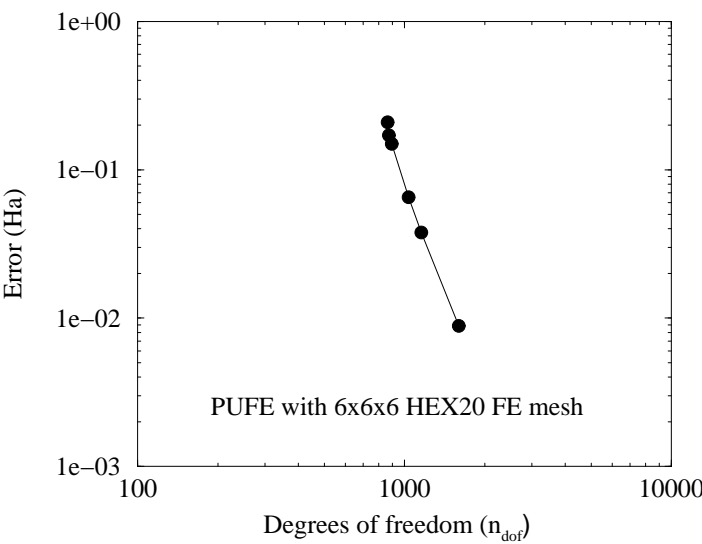

(c)

Figure 5. Error in the sum of the lowest ten eigenvalues for the three-dimensional quantum harmonic oscillator. (a) Higher-order FE; (b),(c) PUFE as a function of the enrichment support radius and the number of degrees of freedom, respectively.

functions

$$
\tilde{\psi}_{n \ell m}(\mathbf{x})=\sum_{\mathbf{R}} \psi_{n \ell m}(|\mathbf{x}-\boldsymbol{\tau}-\mathbf{R}|),
$$

where $\mathbf{R}=i_{1} \mathbf{a}_{1}+i_{2} \mathbf{a}_{2}+i_{3} \mathbf{a}_{3}\left(i_{i}, i_{2}, i_{3}=-2, \ldots, 2\right)$.

The error in the sum of the lowest ten eigenvalues for FE and PUFE solutions is presented Copyright (C) 2008 John Wiley \& Sons, Ltd. Int. J. Numer. Meth. Engng 2008; 00:1-30 
in Figure 5. In Figure 5a, higher-order finite element results as a function of the number of degrees of freedom are shown. The finite element meshes are: $4 \times 4 \times 4,6 \times 6 \times 6,8 \times 8 \times 8$, and $12 \times 12 \times 12$. A $4 \times 4 \times 4$ Gauss quadrature rule is sufficient for cubic FE. From Figure 5a, the rates of convergence in energy eigenvalues for linear, quadratic, and cubic FE are approximately of order $h^{2}, h^{4}$, and $h^{6}$, respectively, consistent with theory. The most refined quadratic mesh yields an error of 0.01261 Ha with 6912 unknowns, whereas the corresponding cubic mesh has an error of 0.00044 Ha with 12096 DOFs. The PUFE results in Figures 5b and 5c are for a $6 \times 6 \times 6$ quadratic FE mesh. The enrichment support radius provides an indication of the extent of enrichment. All nodes that lie within the support radius of the enrichment center $\tau$ are enriched. In Figure 5b, the PUFE results are shown as a function of the enrichment support radius and the same results are depicted in Figure $5 \mathrm{c}$ as a function of the number of degrees of freedom. The benefits of enrichment are striking: for a $6 \times 6 \times 6$ mesh (864 DOFs), the error using quadratic FE is $0.20913 \mathrm{Ha}$, whereas the best PUFE solution on the same mesh has an error of 0.00884 Ha with 1594 DOFs (see Figure 5b).

4.2.2. Periodic Gaussian potential The external potential, $V(\mathbf{x})$, within the unit cell is comprised of contributions from all cells in the infinite lattice of the model Gaussian potential, $V_{g}(r)[23,24]:$

$$
V_{g}(r)=V_{0} \exp \left(-r^{2} / \sigma^{2}\right)
$$

with $V_{0}=-10$ and $\sigma=9.5$ 1.5. The primitive lattice vectors and the potential center are as in (26) with $a=4$ bohr. To compute $V(\mathbf{x})$, second nearest-neighbor cell contributions are sufficient to reproduce the infinite sum:

$$
V(\mathbf{x})=\sum_{\mathbf{R}} V_{g}(|\mathbf{x}-\boldsymbol{\tau}-\mathbf{R}|)
$$

Copyright (c) 2008 John Wiley \& Sons, Ltd.

Int. J. Numer. Meth. Engng 2008; 00:1-30

Prepared using nmeauth.cls 
where the lattice translation vectors $\mathbf{R}=i_{1} \mathbf{a}_{1}+i_{2} \mathbf{a}_{2}+i_{3} \mathbf{a}_{3}\left(i_{i}, i_{2}, i_{3}=-2, \ldots, 2\right)$. The enrichment functions are again the infinite box solutions $\psi_{n \ell m}(\mathbf{x})=R_{n \ell}(r) Y_{\ell m}(\theta, \phi)$, where now the radial solutions, $R_{n \ell}(r)$ are obtained by solving the radial Schrödinger equation [22] using a high-precision spectral element solver [25]. The infinite-box eigenvalues obtained by the spectral solver are listed in Table I. The radial solutions are output at discrete points and are strictly localized by multiplying with a $C^{3}$ cut-off function $h\left(r, r_{0}\right)$ :

$$
h\left(r, r_{0}\right)=\left\{\begin{array}{ll}
1+\frac{20 r^{7}}{r_{0}^{7}}-\frac{70 r^{6}}{r_{0}^{6}}+\frac{84 r^{5}}{r_{0}^{5}}-\frac{35 r^{4}}{r_{0}^{4}}, & r \leq r_{0} \\
0, & r>r_{0}
\end{array},\right.
$$

where $r_{0}=10$ is chosen. A quintic spline-fit of the resulting product is formed, which is then used in the numerical computations. For this problem, all the nodes in the domain are enriched, and second nearest-neighbor cell contributions are summed to form

$$
\tilde{\psi}_{n \ell m}(\mathbf{x})=\sum_{\mathbf{R}} f(\mathbf{R}) \psi_{n \ell m}(|\mathbf{x}-\boldsymbol{\tau}-\mathbf{R}|),
$$

where $f(\mathbf{R})=1$ for periodic enrichment and $f(\mathbf{R})=\exp (i \mathbf{k} \cdot \mathbf{R})$ for Bloch-periodic enrichment (Bloch sum).

Table I. Infinite box eigenvalues for Gaussian-well potential.

\begin{tabular}{|c|c|c|c|}
\hline$n$ & $\ell$ & $\varepsilon_{n \ell}$ & Degeneracy $(2 \ell+1)$ \\
\hline 0 & 0 & -5.958915108 & 1 \\
0 & 1 & -3.587373525 & 3 \\
1 & 0 & -1.874722374 & 1 \\
0 & 2 & -1.522251678 & 5 \\
\hline
\end{tabular}

The results for $\mathbf{k}=[0.12,0.23,0.34]$ (reciprocal lattice coordinates) using three different Copyright (c) 2008 John Wiley \& Sons, Ltd. Int. J. Numer. Meth. Engng 2008; 00:1-30 


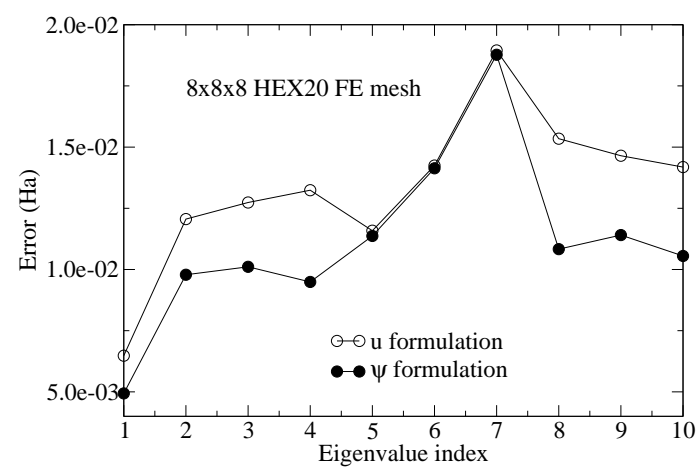

(a)

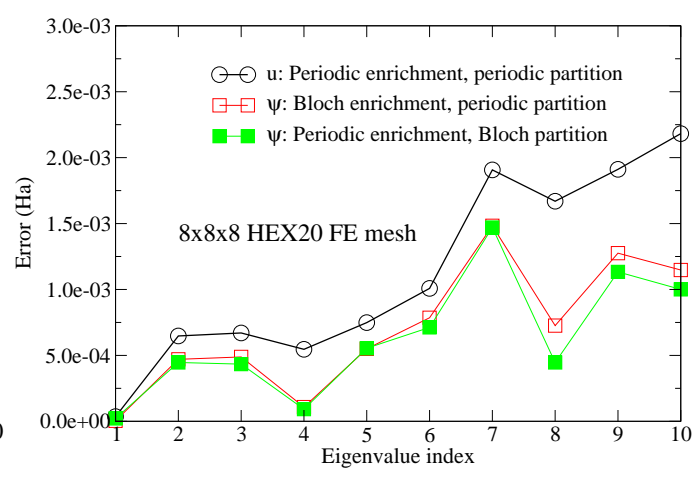

(b)

Figure 6. Error in the lowest ten eigenvalues for the periodic Gaussian-well potential at $\mathbf{k}=[0.12,0.23,0.34]$ using quadratic elements. (a) Bloch FE results for the $u$ formulation and the $\psi$ formulation (see text); and (b) PUFE results for three different Bloch formulations in which the lowest $s$-state and next $p_{y}$-state are enriched.

approaches to impose Bloch-periodic boundary conditions are shown in Figure 6. The approach of Pask et al. [13], which reformulates the Bloch-periodic problem for $\psi$ as an equivalent periodic problem for the associated function $u$, is labeled as the $u$ formulation. The approach introduced here, which solves the Bloch-periodic problem for $\psi$ directly is labeled the $\psi$ formulation. The results are presented for a $8 \times 8 \times 8$ HEX20 finite element mesh $(5 \times 5 \times 5$ Gauss quadrature) that has 2048 DOFs. It is observed from Figure 6a that for finite elements, the $\psi$ formulation provides consistently better accuracy than the $u$ formulation. In the PUFE studies, two enrichment functions are used: the lowest $s$-state and the next $p_{y}$-state are enriched. There are 3073 DOFs in the PUFE analyses, and $8 \times 8 \times 8$ Gauss quadrature is used. From the plots, the dramatic improvements of PUFE vis-à-vis FE are revealed. A typical requirement in quantum mechanical calculations is $10^{-3} \mathrm{Ha}$ /atom or better accuracy in energies. In the PUFE solutions, the lowest eigenvalue and the fourth eigenvalue correspond 
to states that are enriched, and these eigenvalues are computed to $\mathcal{O}\left(10^{-5}\right)$ accuracy. As discussed in Section 3.2, there are two distinct ways of forming a Bloch-periodic PUFE basis: (1) Bloch-periodic enrichment with periodic partition of unity; or (2) periodic enrichment with Bloch-periodic partition of unity (which is then not strictly a partition of unity). The results of these two $\psi$-based formulations are shown along with those of the corresponding $u$-based formulation employing the same periodic enrichment as in the $\psi$-based formulation with Bloch-periodic partition. The results demonstrate two key findings. First, enrichment is more effective in the $\psi$ formulation than in the $u$ formulation, as evidenced by the marked relative improvement of the 1 st and 4 th eigenvalues in both $\psi$ formulations. Second, while computationally far less expensive in the context of numerous k-points, the $\psi$ formulation with fixed periodic enrichment is as good or better than the $\psi$ formulation with Bloch-periodic enrichment, which must be recomputed via Bloch sum at each new k-point.

\section{CONCLUDING REMARKS}

In this paper, we developed classical and partition-of-unity enriched finite element formulations to impose Bloch-periodic boundary conditions. To this end, we considered the quantummechanical problem in a crystalline solid, which consists of the solution of the Poisson and Schrödinger equations in a parallelepiped unit cell subject to periodic and Bloch-periodic boundary conditions, respectively. The weak forms for these equations were derived, with particular attention to the imposition of both value- and derivative-periodic parts of the required boundary conditions. The construction of periodic and Bloch-periodic finite element and enriched bases was presented, and two variationally consistent approaches to obtain the corresponding system matrices were derived: one via modification of the element connectivity 
matrices; the other, via row and column operations on the standard Neumann matrices.

Numerical examples for the Poisson and Schrödinger equations using classical and enriched higher-order finite elements were presented. For the Poisson problem subject to periodic boundary conditions, a suitable periodic enrichment function was constructed. The optimal rate of convergence in strain energy for higher-order FE was attained. The enriched quadratic FE solution also had the optimal convergence rate and was about two orders of magnitude more accurate than the corresponding FE solution. For the Schrödinger equation, the threedimensional harmonic oscillator in a finite box subject to periodic boundary conditions $(\mathbf{k}=\mathbf{0})$ was treated using FE and PUFE methods. The optimal convergence rate of the energy eigenvalues for higher-order FE was established. Enrichment of the ten lowest eigenstates led to marked improvements in accuracy on a coarse mesh with the addition of only a modest number of degrees of freedom. The Gaussian-well problem for $\mathbf{k} \neq \mathbf{0}$ was solved to demonstrate the enforcement of Bloch-boundary conditions in FE and PUFE methods. Different schemes for imposing general Bloch boundary conditions were explored, and it was found that the new scheme ( $\psi$ formulation) developed here was more accurate than previous Bloch- $u$ based formulations, both with and without enrichment. The proposed algorithm to impose Blochperiodic boundary conditions in finite elements has a sound variational basis, and provides a systematic means to treat such boundary conditions in the modeling of wave-like phenomena using finite element and enriched finite element methods.

\section{ACKNOWLEDGMENTS}

This work was performed under the auspices of the U.S. Department of Energy by Lawrence Livermore National Laboratory under Contract DE-AC52-07NA27344.

Copyright (C) 2008 John Wiley \& Sons, Ltd.

Int. J. Numer. Meth. Engng 2008; 00:1-30

Prepared using nmeauth.cls 


\section{REFERENCES}

1. J. C. Michel, H. Moulinec, and P. Suquet. Effective properties of composite materials with periodic microstructure: a computational approach. Comput. Meth. Appl. Mech. Engng., 172:109-143, 1999.

2. R. Porter and D. Porter. Scattered and free waves over periodic beds. J. Fluid Mech., 483:129-163, 2003.

3. E. Yablonovitch. Photonic band-gap crystals. J. Phys.: Condens. Matter, 5:2443-2460, 1993.

4. B. P. Hiett, J. M. Generowicz, S. J. Cox, M. Molinari, D. H. Beckett, and K. S. Thomas. Application of finite element methods to photonic crystal modelling. IEE Proc - Sci. Meas. Technol., 149(5):293-296, 2002.

5. A. Nicolet, S. Guenneau, C. Geuzaine, and F. Zolla. Modelling of electromagnetic waves in periodic media with finite elements. J. Comp. Appl. Math., 168(1-2):321-329, 2004.

6. N. W. Ashcroft and N. D. Mermin. Solid State Physics. Holt, Rinehart and Winston, New York, 1976.

7. J. B. Davies, F. A. Fernandez, and G. Y. Philippou. Finite-element analysis of all modes in cavities with circular symmetry. IEEE Trans. Microw. Theory Tech., 30:1975-1980, 1982.

8. D. T. McGrath and V. P. Pyati. Phased array antenna analysis with the hybrid finite element method. IEEE Trans. Antennas Propag., 42(12):1625-1630, 1994.

9. D. T. McGrath and V. P. Pyati. Periodic structure analysis using a hybrid finite element method. Radio Sci., 31(5):1173-1179, 1996.

10. C. Mias, J. P. Webb, and R. L. Ferrari. Finite element modelling of electromagnetic waves in doubly and triply periodic structures. IEE Proc. - Optoelectron., 146:111-118, 1999.

11. B. Hermansson and D. Yevick. Finite-element approach to band-structure analysis. Phys. Rev. B, 33(10):7241, 1986.

12. R. L. Ferrari. Electronic band structure for two-dimensional periodic lattice quantum configurations by the finite element method. Int. J. Numer. Model. Electron. Netw., Devices, Fields, 6:283-297, 1993.

13. J. E. Pask, B. M. Klein, C. Y. Fong, and P. A. Sterne. Real-space local polynomial basis for solid-state electronic-structure calculations: A finite-element approach. Phys. Rev. B, 59(19):12352, 1999.

14. J. E. Pask, B. M. Klein, P. A. Sterne, and C. Y. Fong. Finite-element methods in electronic-structure theory. Comput. Phys. Commun., 135(1):1-34, 2001.

15. J. E. Pask and P. A. Sterne. Finite element methods in ab initio electronic structure calculations. Model. Simul. Mater. Sci. Eng., 13(3):R71-R96, 2005.

16. S. Jun. Meshfree implementation for the real-space electronic-structure calculation of crystalline solids.

Copyright (c) 2008 John Wiley \& Sons, Ltd.

Int. J. Numer. Meth. Engng 2008; 00:1-30

Prepared using nmeauth.cls 
Int. J. Numer. Meth. Engng., 59(14):1909-1923, 2004.

17. G. Strang and G. Fix. An Analysis of the Finite Element Method. Prentice-Hall, Englewood Cliffs, N.J., 1973.

18. O. C. Zienkiewicz, R. L. Taylor, and J. Z. Zhu. The Finite Element Method: Its Basis and Fundamentals. Elsevier Butterworth-Heinemann, Burlington, MA, sixth edition, 2005.

19. J. M. Melenk and I. Babuška. The partition of unity finite element method: Basic theory and applications. Comput. Meth. Appl. Mech. Engng., 139:289-314, 1996.

20. I. Babuška and J. M. Melenk. The partition of unity method. Int. J. Numer. Meth. Engng., 40:727-758, 1997.

21. G. B. Arfken and H.-J. Weber. Mathematical Methods for Physicists. Elsevier, Boston, sixth edition, 2005.

22. D. J. Griffiths. Introduction to Quantum Mechanics. Prentice Hall, Englewood Cliffs, 1994.

23. F. Gygi. Adaptive Riemannian metric for plane-wave electronic-structure calculations. Europhys. Lett., 19(7):617-622, 1992.

24. E. Tsuchida and M. Tsukada. Adaptive finite-element method for electronic-structure calculations. Phys. Rev. B, 54(11):7602, 1996.

25. J. E. Pask and N. Sukumar. Spectral element method for quantum mechanical atomic calculations. 2008. in preparation. 